\title{
Performance of the MRPC-based Time-Of-Flight detector of ALICE at LHC
}

\author{
Andrea Alici* (for the ALICE Collaboration) \\ Museo Storico della Fisica e Centro Studi e Ricerche "Enrico Fermi", Rome, Italy \\ Dipartimento di Fisica dell'Universitá and Sezione INFN, Bologna, Italy \\ E-mail: alici@bo.infn.it
}

The large Time-Of-Flight (TOF) array is one of the main detectors devoted to charged hadron identification in the mid-rapidity region of the ALICE experiment at the LHC. It allows separation among pions, kaons and protons up to a few $\mathrm{GeV} / \mathrm{c}$, covering the full azimuthal angle and -0.9 $<\eta<0.9$. The TOF exploits the innovative MRPC technology capable of an intrinsic time resolution better than $50 \mathrm{ps}$ with an efficiency close to $100 \%$ and a large operational plateau; the full array consists of 1593 MRPCs covering a cylindrical surface of $141 \mathrm{~m}^{2}$. In this report, the TOF performance during the 2010 and 2011 data taking periods is reported together with selected physics results obtained with $\mathrm{pp}$ and $\mathrm{Pb}-\mathrm{Pb}$ collisions.

XI workshop on Resistive Plate Chambers and Related Detectors - RCP2012,

February 5-10, 2012

INFN Laboratori Nazionali di Frascati Italy

* Speaker. 


\section{Introduction}

ALICE [1] (A Large Ion Collider Experiment) is the dedicated heavy-ion experiment at the CERN LHC designed to address the physics of strongly interacting matter and the quark-gluon plasma at extreme values of energy density. ALICE is also studying proton-proton collisions both for comparison with $\mathrm{Pb}-\mathrm{Pb}$ collisions and in physics areas where ALICE is complementary with other LHC experiments. Particle IDentification (PID) is a crucial aspect of the ALICE experiment and it is performed by combining different detecting systems [2].

The Time-Of-Flight (TOF) system [3] is devoted to charged hadron identification in the midrapidity region. It aims at $3 \sigma \pi / K$ and $K / p$ separation for momenta up to $2.5 \mathrm{GeV} / \mathrm{c}$ and $4 \mathrm{GeV} / \mathrm{c}$ respectively; in order to reach this goal the TOF must provide a time resolution better than $100 \mathrm{ps}$. The TOF is a large area detector covering a cylindrical surface of $141 \mathrm{~m}^{2}$ with an inner radius of $3.7 \mathrm{~m}$, a pseudorapidity interval $[-0.9,+0.9]$ and full azimuthal coverage. The TOF exploits the innovative Multigap Resistive Plate Chamber (MRPC) [4] technology, capable of an intrinsic time resolution better than $50 \mathrm{ps}$ with an efficiency close to $100 \%$ and a large operational plateau. The whole system is made of 1593 MRPCs arranged into 90 gas-tight modules which are grouped into 18 SuperModules (SM) each covering an azimuthal angle of 20 degrees.

\section{Design of the ALICE TOF Multigap RPC}

The MRPCs for the ALICE TOF detector are designed as double-stack strips of $7.4 \times 120$ $\mathrm{cm}^{2}$ active area. Each stack has 5 gas gaps of $250 \mu \mathrm{m}$ width; the two stacks are placed on each side of a central anode. The resistive plates are made of high-resistivity $\left(\approx 10^{13} \Omega \mathrm{cm}\right)$ soda-lime commercial glass $0.4 \mathrm{~mm}$ thick. The glass plates have been chosen to ensure the high uniformity of the gap size needed to achieve a very high time resolution; however the glass demonstrated also a good stability for long-term behavior. External electrodes are obtained from $550 \mu \mathrm{m}$ thick glasses; the high voltage is applied on the outer surface of this plates by means of a specially developed acrylic paint loaded with metal oxides giving a surface resistivity between 2 and $25 \mathrm{M} \Omega / \square$. The pick-up elements are $2.5 \times 3.5 \mathrm{~cm}^{2}$ area pads (96 per strip) placed outside the external plates and arranged in two-row arrays of 48 pads; the choice of this area for the readout pads was driven by the request to limit the occupancy at the $15 \%$ level even in the case of $\mathrm{Pb}-\mathrm{Pb}$ collisions where some $10^{3}$ primary charged particles per unit of rapidity were expected. The applied electric field is very high, greater than $100 \mathrm{kV} / \mathrm{cm}$. For these values, the growth of the avalanche in the gas volume is dominated by space-charge effects and the MRPC can be operated in saturated avalanche mode; the average charge produced by a through-going particle is at the level of $2-3 \mathrm{pC}$.

The gas mixture used in the ALICE experiment is a non-flammable mixture composed by $93 \%$ of $\mathrm{C}_{2} \mathrm{H}_{2} \mathrm{~F}_{4}$ and $7 \%$ of $\mathrm{SF}_{6}$.

Test beam results may be found in [5, 6], where a time resolution better than $50 \mathrm{ps}$, including all of the electronic readout contribution, and an efficiency close to $100 \%$ with a long streamer-free efficiency plateau have been measured.

\section{Rate capability, ageing and efficiency}

The rate capability of the MRPCs has been checked with dedicated tests at the Gamma Ir- 


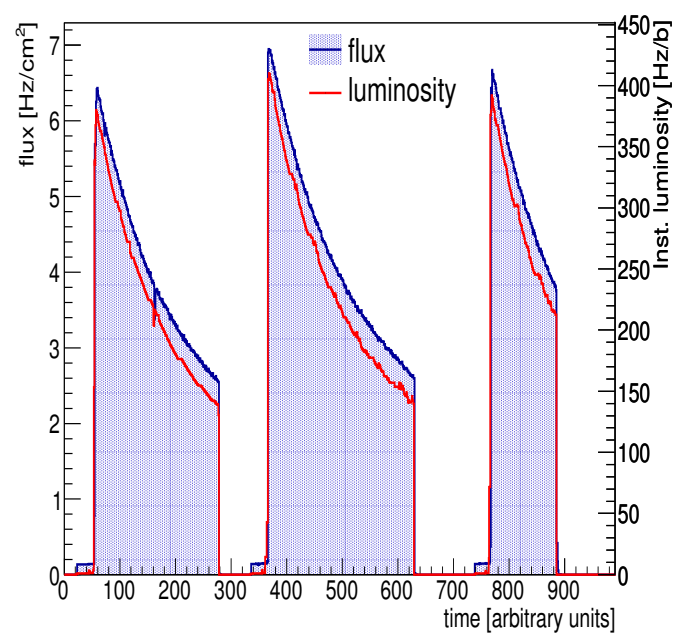

Figure 1: Flux of charged particles at TOF and ALICE instantaneous luminosity as a function of time during the 2011 LHC heavy-ion runs.

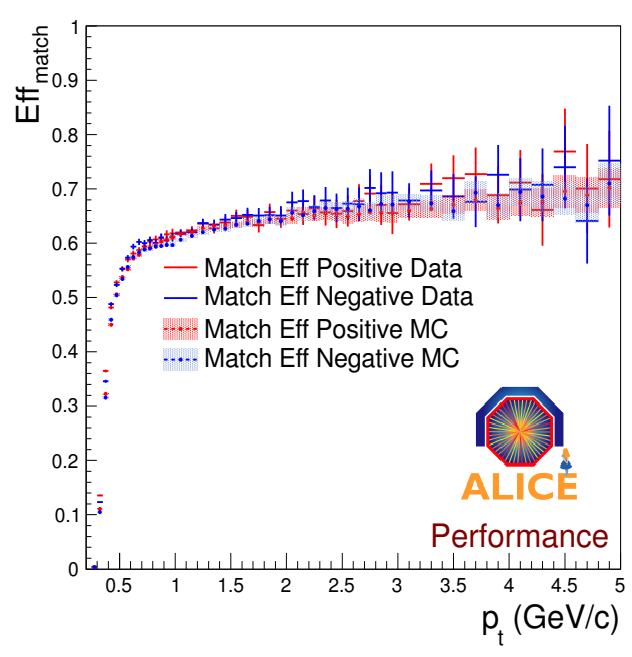

Figure 2: TOF track matching efficiency, as a function of $p_{t}$, compared for positive and negative particles in real data and in MC.

radiation Facility (GIF) at CERN [7, 8]; these tests demonstrated that the MPRCs designed for the ALICE TOF can operate far in excess of the $50 \mathrm{~Hz} / \mathrm{cm}^{2}$, which is the maximum rate expected at TOF assuming a charged particle density of $\mathrm{dN}_{c h} / \mathrm{d} \eta=8000$ for central $\mathrm{Pb}-\mathrm{Pb}$ collisions and $L=10^{27} \mathrm{~cm}^{-2} \mathrm{~s}^{-1}$ of luminosity. The main reason is the small amount of charge produced for each through-going particle. However, from analysis of $2010 \mathrm{~Pb}-\mathrm{Pb}$ data at $\sqrt{s_{N N}}=2.76 \mathrm{TeV}$ a particle density of $\mathrm{dN}_{c h} / \mathrm{d} \eta=2000$ can be assumed. In Fig. 1 the estimated flux of charged particles at TOF and the instantaneous luminosity in ALICE are showed as a function of time. The flux has been estimated by multiplying the measured single trigger-pad rate (each trigger-pad covers 888 $\mathrm{cm}^{2}$ equivalent to the area of one MRPC) for the measured average number of hits per trigger-pad. Before the ramp of the luminosity, a small dark rate of $0.1-0.2 \mathrm{~Hz} / \mathrm{cm}^{2}$ is observed. After the ramp, the TOF single trigger-pad rate is proportional to the luminosity. The estimated flux of charged particles is $\approx 7 \mathrm{~Hz} / \mathrm{cm}^{2}$ for a luminosity of $L=4 \cdot 10^{26} \mathrm{~cm}^{-2} \mathrm{~s}^{-1}$.

The current drawn by the full detector for these values of the luminosity is at the level of a few tens of $\mu \mathrm{A}$ for 1593 MRPCs, less than 25nA per MRPC. By dividing the current for the flux of charged particles we get an average charge produced inside the MRPC by a through-going particle of the order of $3 \mathrm{pC}$; this is the same value measured during the tests with beam of charged particles in the R\&D phase with MRPCs from the mass production. It is an indication that no ageing effects are present in the MRPC, as already pointed out during dedicated tests $[8,9]$.

The small current and the low noise rate allow to use the TOF as a trigger detector in ALICE. Thus, the TOF trigger is the preferred choice for cosmic runs in ALICE, with a rate around $10 \mathrm{~Hz}$ for pure back-to-back topology and 50\% of purity [10]. These results have been achieved running all the MRPCs at the same voltage and with all front-end electronic thresholds set at the same value. During the 2010 and 2011 LHC heavy-ion program the TOF contributed also to the trigger of Ultra-Peripheral Collisions (UPC) in the barrel.

Also the MRPCs efficiency appears to be stable over time. In Fig. 2 the TOF track matching 
efficiency, defined as the ratio between the number of tracks reconstructed in the TPC that have produced a detectable signal on TOF and the total number of tracks reconstructed in the TPC, is compared for positive and negative particles in a Monte Carlo (MC) sample and with pp collisions recorded in 2010 for different $p_{t}$ values. To be noted that the matching efficiency includes not only the MRPC efficiencies but also dead spaces in the TOF array, particle decays, interaction with materials as well as inefficiencies of the algorithm. In the MC the single pad efficiency is $98 \%$, averaged over the whole pad area and as measured in the test beam. The good match between MC and real data, for this and for other particle samples, indicates that the TOF efficiency is similar to the $98 \%$ used in the MC simulations, of the same order of that measured during the MRPC mass production.

\section{TOF PID performance}

A time-of-flight measurement yield the velocity of a charged particle by measuring the particle flight time $t$ over a given distance along the track trajectory $L$; the particle flight time can be expressed as $t=$ time $_{\text {hit }}-t_{0}$, where time $_{h i t}$ is the time measurement made by the TOF detector while $t_{0}$ is the time of the interaction, measured in ALICE by means of:

A. a dedicated Cherenkov detector (T0);

B. TOF detector itself if the number of tracks reaching the TOF is large enough $(\geq 3)$ to perform a global time minimization;

C. the average $t_{0}$ of LHC fill, if A or B are not available (on an event-by-event basis).

A measurement of the TOF global time resolution can be performed by plotting the distribution of the difference between measured and expected time of arrival of pions on TOF and by fitting it with a gaussian function, as in Fig. 3 where pion candidates have been selected by TPC $d E / d x$ measurements. In this plot TOF means the time measured by the TOF detector while TOF $(\pi, \exp )$ is the expected time of arrival on TOF and it is computed during reconstruction by ALICE core central tracking; the red curve shows the gaussian fit to the data. The plot has been obtained with $\mathrm{Pb}-\mathrm{Pb}$ collisions at $\sqrt{s_{N N}}=2.76 \mathrm{TeV}$ recorded in November 2010. The result of the fit shows a TOF time resolution of $86 \mathrm{ps}$; the same results are obtained for 2011 data. The value quoted here includes the intrinsic time resolution of the detector technology plus contributions from electronics (amplification and time-to-digital conversion), uncertainties arising from the distribution of the digital clock from the LHC to the experiments, the spread of the $t_{0}$ signal (here $\approx 10$ ps obtained with method B), contributions from the tracking and from calibration. In future the latter can be improved by:

- single channel time-slewing corrections (finite rise time of the amplifying electronics);

- time-walk corrections (signal propagation delays on the pick-up pad).

In Fig. 4 the achieved separation for $\pi / K$ and $K / p$ as a function of transverse momentum in TOF is shown. A two sigma separation is achieved up to $3 \mathrm{GeV}$ for $\pi / K$ and up to $5 \mathrm{GeV}$ for $K / p$ 


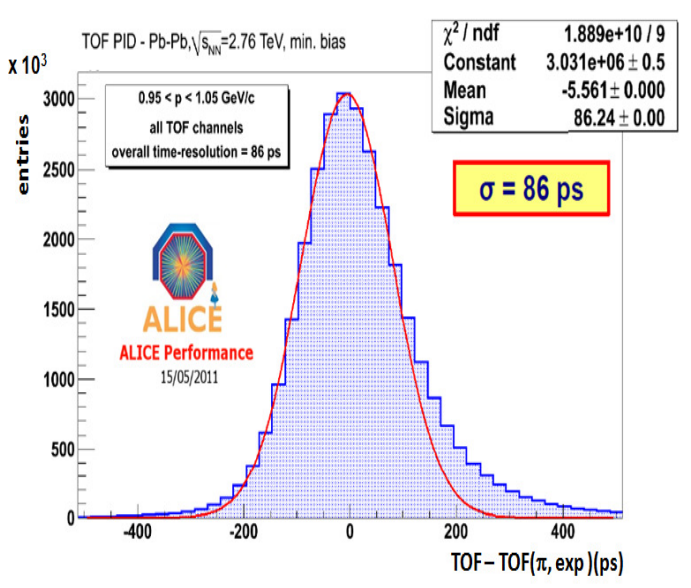

Figure 3: Distribution of the difference between measured and expected time of arrival on TOF for selected pions. The red curve shows the gaussian fit to the data.

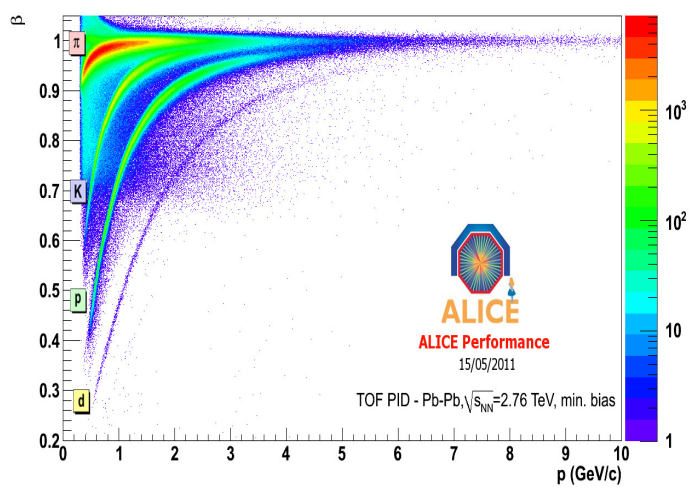

Figure 5: TOF measured particle $\beta$ vs. momentum for $\mathrm{Pb}-\mathrm{Pb}$ collisions at $\sqrt{s_{N N}}=2.76 \mathrm{TeV}$.

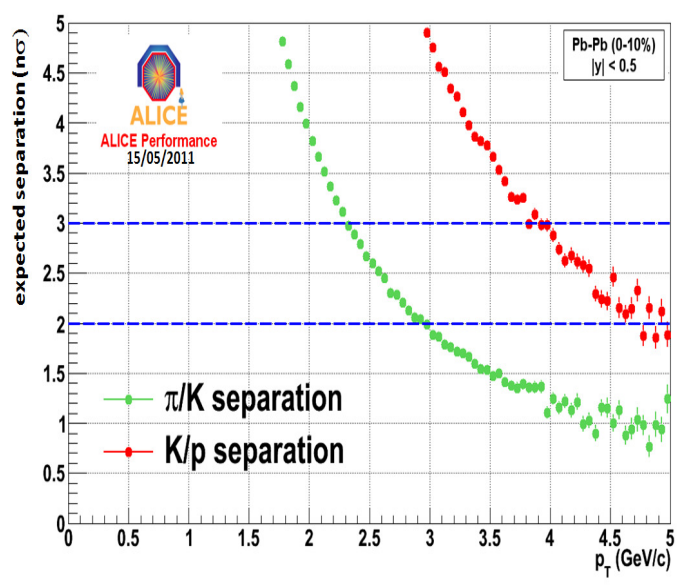

Figure 4: Expected separation (nsigma) for $\pi / K$ and $K / p$ as a function of transverse momentum in TOF. Green points $<\left(t_{K, \text { exp }}-t_{\pi, \text { exp }}\right) / \sigma(K)>$, red points $<\left(t_{p, \exp }-t_{K, \exp }\right) / \sigma(p)>$.

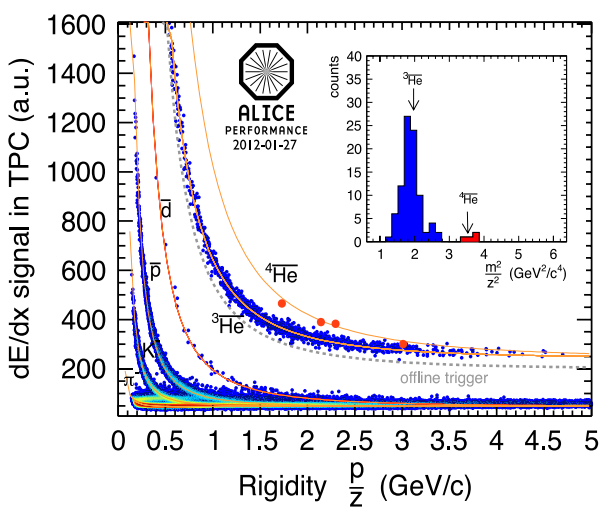

Figure 6: Four anti-alpha candidates (indicated in red) found in ALICE in 16 million $\mathrm{Pb}$ - $\mathrm{Pb}$ events. The insert shows the combined mass measurement with the TOF system.

even with a still non-optimal calibration. The plot is based on expected integrated times at TOF smeared according experimental resolution achieved in $\mathrm{Pb}-\mathrm{Pb}$ central collisions for the different contributions.

In Fig. 5 a plot of the TOF-measured $\beta$ as a function of momentum, obtained in $\mathrm{Pb}-\mathrm{Pb}$ collisions at $\sqrt{s_{N N}}=2.76 \mathrm{TeV}$ is shown. The different particle species are clearly visible.

\section{Analysis with TOF}

The TOF information is an important part of the PID analysis which is a distinguished feature of the ALICE experiment. Here follow a few examples.

Fig. 6 shows the TPC $d E / d x$ vs rigidity distribution for all events passing the offline trigger 
selection of events with at least one $\mathrm{Z}>1$ track candidate; only negatively charged particles are shown. Above a rigidity of $p / z=2.3 \mathrm{GeV} / \mathrm{c}$ however the $d E / d x$ bands from ${ }^{3} \overline{\mathrm{He}}$ and ${ }^{4} \overline{\mathrm{He}}$ are overlapping and the mass calculated with the TOF is needed to separate these two species. The insert shows the combined mass measurement with the TOF system which is in particular needed to separate the candidate with the largest momentum from the ${ }^{3} \overline{H e}$ line.

Moreover the TOF information allows us:

i to extend the $p_{t}$ reach of the spectra and the analysis of the anisotropic flow of identified hadrons;

ii to identify weak decays of strange particles with a sufficiently long lifetime via their characteristic decay topology;

iii to improve the separation between electrons and pions in the TPC, by removing the slower hadrons, for a given momentum, with a cut on the particle velocity.

\section{Conclusion}

The ALICE TOF detector has been taking data in 2010 and 2011 LHC physics runs with high performance in terms of dark current, noise, efficiency and time resolution. No evidences of ageing effects, as increase of dark current or performance degradation, have been observed after two years of data taking, confirming the results of ageing tests carried out during the R\&D phase which have clearly indicated that ageing is not an issue for the MRPCs designed for the ALICE TOF.

During the 2010 and 2011 data taking the TOF successfully provided particle identification both for $\mathrm{pp}$ and $\mathrm{Pb}-\mathrm{Pb}$ collisions and many analysis carried out by the ALICE Collaboration are widely using information provided by the TOF detector.

\section{References}

[1] ALICE Collaboration, J. Phys. G: Nucl. Part. Phys. 30 (2004) 1517; J. Phys. G: Nucl. Part. Phys. 32 (2006) 1295; IOP-JINST 3 (2008) S08002.

[2] ALICE Collaboration, ALICE: physics performance report, volume II, J. Phys. G32 (2006) 1295-2040.

[3] ALICE Collaboration, Addendum to TOF Technical Design Report, CERN/LHCC 2002-016.

[4] E. Cerron-Zeballos et al., Nucl. Instrum. Meth. A374, 132 (1996).

[5] A. Akindinov et al, Nucl. Instrum. Meth. A532, 611-621 (2004).

[6] A. Akindinov et al, Nuovo Cimento B124, 235-253 (2009).

[7] A. Akindinov et al., Nucl. Instrum. Meth. A490, 58-70 (2002).

[8] A. Alici et al., Nucl. Instrum. Meth. A579, 979-988 (2007).

[9] A. Akindinov et al., Nucl. Instrum. Meth. A533, 93-97 (2004).

[10] A. Akindinov et al, Eur. Phys. J. C68, 89-108 (2010). 